\title{
Development of bronchiectasis during long-term rituximab treatment for rheumatoid arthritis
}

The authors declare no financial disclosure

\begin{abstract}
Here are the cases of three female patients who received long-term rituximab treatment for seropositive, erosive and deforming rheumatoid arthritis were reported. After rituximab treatment, they presented with recurrent sinusitis and pneumonia, followed by the subsequent development of bronchiectasis. A temporal relationship between rituximab treatment and the onset of respiratory complications was exposed as a possible pathogenic mechanism.
\end{abstract}

Key words: bronchiectasis, rheumatoid arthritis, rituximab, sinusitis

Adv Respir Med. 2018; 86: 310-313

\section{Introduction}

Rituximab is a chimeric monoclonal antibody that targets the transmembrane CD20, which induces the ablation of mature pre-B and B lymphocytes [1]. It was approved by the Food and Drug Administration for the treatment of non-Hodgkin's lymphoma (1997), rheumatoid arthritis (RA) (2006), and positive ANCA vasculitis, such as microscopic polyangiitis and granulomatosis with polyangiitis (2011) [2]. Other indications for rituximab treatment in autoimmune diseases include systemic lupus erythematosus and inflammatory myopathies [3, 4]. The most relevant adverse events are related to the infusion of rituximab, and less frequently, immunological, respiratory, renal, cardiac and haematological disorders as well as the increase of neoplasms and infections. Pulmonary adverse reactions occur in $5.3 \%$ of patients undergoing rituximab treatment [5], including interstitial lung disease [6], asthma, bronchiolitis obliterans, hypersensitivity pneumonitis, and diffuse alveolar haemorrhage [7-9]. A case of a 17-year-old patient who was receiving $1000 \mathrm{mg}$ of rituximab every 6 months for 6 years for optic neuromyelitis was recently published; the patient developed rhinosinusitis and recurrent pneumonia with the development of bronchiectasis in the context of hypogamaglobulinaemia [10].

\section{Case reports}

In a cohort of 964 patients with RA treated since 2007 at the Fundación Valle del Lili, a referral care centre in Southwest Colombia, 164 (17\%) received biological therapy based on the institutional treatment guidelines. Eighty-four patients (8.7\%) were given rituximab. The average age at the onset of the disease was 34 (range: 17-60, SD 15). Indications for the initiation of rituximab treatment were failure to respond to anti-TNF in 45 (53\%) patients, failure to respond to methotrexate in $27(32 \%)$ and other reasons in 12 (14\%). The average number of rituximab cycles was 4 . Each cycle comprised a $1000 \mathrm{mg}$ initial dose and followed with $1000 \mathrm{mg}$ at 2 weeks, indicated for 9 months. There was a lack of therapeutic effec- 
tiveness in six patients (7\%). In addition to the de novo development of bronchiectasis, recurrent sinusitis and pneumonias were present in three $(3.5 \%)$ subjects.

\section{Case 1}

A 76-year-old woman presenting with seropositive, erosive and deforming RA, which initiated at the age of 58, visited our centre with a history of chronic obstructive pulmonary disease (COPD) secondary to smoking manifesting with a chronic, productive cough. A chest CT from 2013 is shown in Figure 1A. She received methotrexate, leflunomide, etanercept (2008-2011) and tocilizumab (2011-2013) treatment with a notable loss of effectiveness. Rituximab was started with a good response in 2013. Two months after the fourth cycle of rituximab, she presented with pansinusitis and multilobar pneumonia, requiring hospital treatment with parenteral antibiotics. Three months after this episode, she developed a lower respiratory infection with residual cough and a progressive increase in expectoration. CT of the thorax was performed (Fig. 1B), which revealed de novo basal cylindrical bronchiectasis. Liver and kidney function tests and serum gammaglobulin levels were normal. Because it was suspected that rituximab played a role in the development of this pulmonary disease, it was decided to withdraw rituximab and initiate oral steroids and tofacitinib treatment. The patient required chronic respiratory therapy to control bronchiectasis symptoms.

\section{Case 2}

A 63-year-old woman with occupational exposure to volatile solvents, dust and boiler smoke underwent treatment for seropositive, erosive and deforming RA since the age of 48 . She presented with additional conditions, including hypothyroidism and kidney stones, and denied smoking. She presented refractoriness to the treatment of RA with conventional medications (prednisolone, methotrexate and hydroxychloroquine). Rituximab was started in 2009. After the fifth cycle of rituximab, she presented with recurrent high and low respiratory infections (three episodes) that required hospital treatments. A chest CT scan from 2013, which is shown in Figure 1C, revealed mild chronic bronchitic changes. Since then, the patient has reported productive and progressive cough, as well as episodes of dyspnoea associated with bronchospasm. Chronic inhalers with steroids, bronchodilators, respiratory therapy and the management of recurrent infections with antibiotic therapy were indicated. A more recent chest CT scan from 2017 showed cylindrical bronchiectasis in both lower lobes (Fig. 1D), and a CT scan of the paranasal sinuses suggested chronic sinus disease. CBC, liver and kidney functions and serum gammaglobulin levels were normal. RA is currently being managed with tofacitinib and low doses of oral steroids.

\section{Case 3}

A 65-year-old woman presented with seropositive, erosive and deforming RA, which initiated at the age of 48. She had a history of exposure to wood smoke and cigarettes. She was treated for several years with prednisolone, methotrexate and chloroquine. In 2006, etanercept was indicated for refractory disease. However, she developed drug-induced lupus (DIL) with glomerulonephritis and cutaneous involvement. Subsequently, etanercept was withdrawn, and rituximab was initiated for both DIL and RA control [11]. After the sixth cycle of rituximab, she complained of headache, rhinorrhoea, cough of progressive intensity, expectoration and dyspnoea. Pansinusitis and the incipient development of bronchiectasis were documented (Fig. 1E). She was hospitalised in 2016 because of worsening symptoms. The chest CT scan on admission is shown in Figure 1f. Klebsiella oxytoca was isolated from orotracheal secretion. Chronic ventilator support was necessary, and tracheostomy was performed. Six weeks after admission, the patient died.

\section{Discussion}

This study reported the cases of three female patients aged $>60$ who received rituximab treatment for the control of seropositive RA refractory to conventional and biological treatments. They were given four, five and six cycles of rituximab, respectively (cycles of $1000 \mathrm{mg}$ every 2 weeks every 9 months) before presenting with recurrent sinusitis and pneumonia and subsequently, bronchiectasis. These three patients had a history of smoking and/or exposure to environmental contaminants. Sinusitis was confirmed via imaging studies, in addition to changes in haematological tests that are typically indicative of bacterial infection and rising acute phase reactants. They presented with cough with high volumes of sputum, which lead to the suspicion of the presence of bronchiectasis, which was confirmed with a chest CT scan. The patients did not show hypogammaglobulinaemia. 


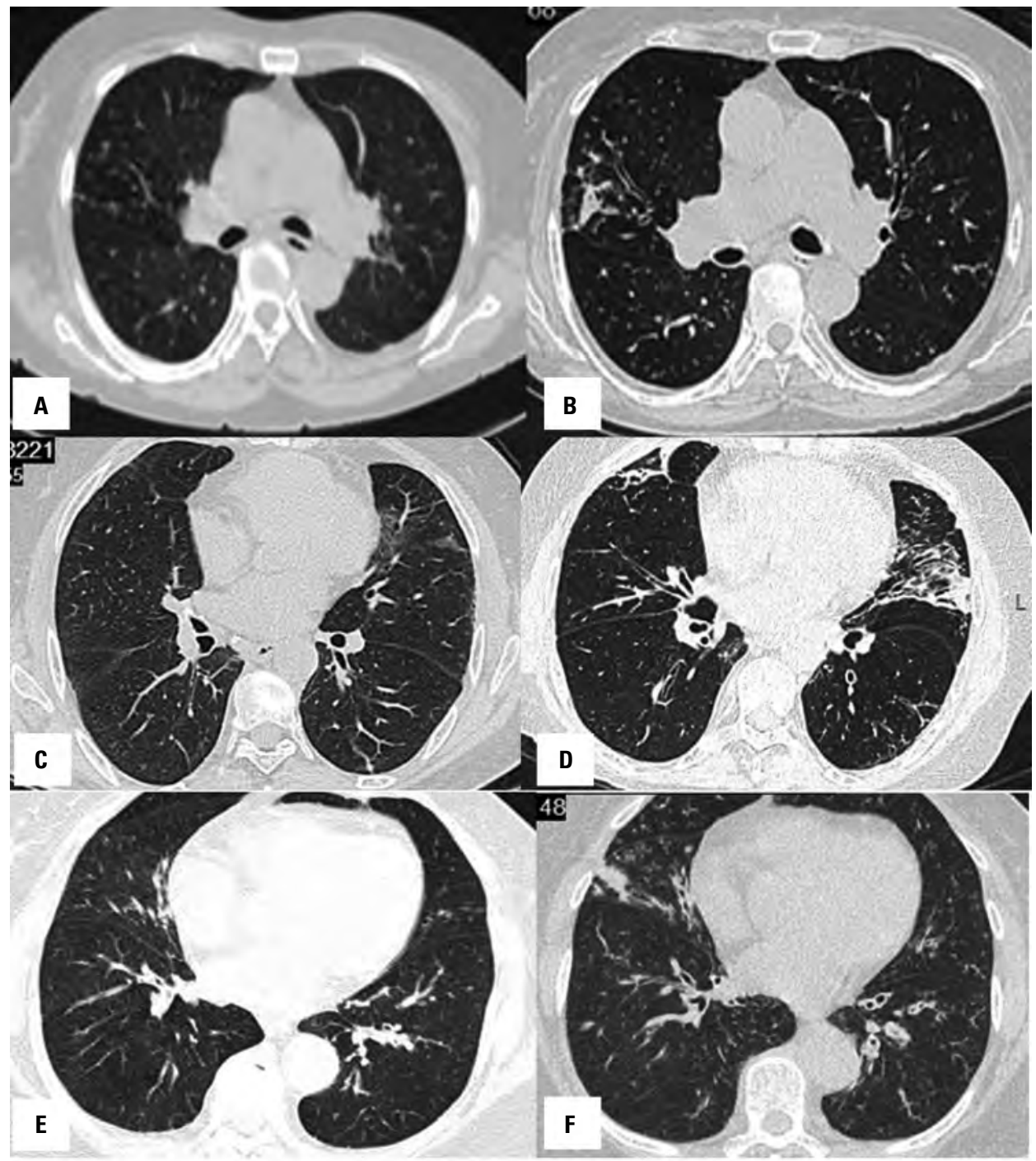

Figure 1. Case 1: A — chest CT from 2013 reported as normal; B — chest CT from 2015 showing cylindrical bronchiectasis in both lung bases. Case 2: C — chest CT from 2013 showing nonspecific bronchial changes; D — chest CT from 2017 showing cylindrical bronchiectasis in both lower lobes. Case 3: E — chest CT from 2014 with incipient bronchiectasis; F — chest CT from 2016 showing the progression of bronchiectasis

Bronchiectasis is a condition wherein the bronchi and bronchioles dilate as a consequence of the damage to their walls [12], which leads to a loss of the mucociliary defence mechanisms with consequent colonisation by microorganisms; this in turn causes chronic inflammation and worsening of the tissues that integrate the bronchi and bronchioles [13-18]. Other conditions that may coexist in the same patient, such as foreign body aspiration, gastro-oesophageal reflux, immune deficiencies, COPD or conditions similar to RA, have previously been associated with the development of bronchiectasis in adults [19]. Rituximab can affect both cellular and humoral immunity [20]. Several of these conditions were present in the three patients in this report.

Pulmonary reactions related to rituximab treatment were reported to have occurred in 5.3\% of patients in a previous study [5]. Prolonged treatment with this drug has been announced to be associated with toxicity in the respiratory system, which was named by Bitzan et al. [6] as 'rituximab (B-cell depleting antibody) associated lung injury (RALI)'. These patients were children who presented with haematological malignancies, configuring a form of interstitial lung disease with reports of lymphocytic infiltrates in their histopathological samples.

The temporal relationship between rituximab treatment and the onset of respiratory complications, such as chronic sinusitis, recurrent pneumonia and the subsequent bronchiectasis, in these three patients suggests a pathogenic mechanism. Age, the presence of severe RA and chronic exposure to air pollutants may be contributing factors that predispose patients to this phenome- 
non. Future studies are needed to corroborate this relationship and to make more precise, preventive or therapeutic recommendations for the use or disuse of long-term rituximab treatment for RA.

\section{Conflict of interest}

The authors declare do conflict of interest.

\section{References:}

1. Taylor RP, Lindorfer MA. Drug insight: the mechanism of action of rituximab in autoimmune disease - the immune complex decoy hypothesis. Nat Clin Pract Rheumatol. 2007; 3(2): 86-95, doi: 10.1038/ncprheum0424, indexed in Pubmed: 17299446.

2. Storz U. Rituximab: how approval history is reflected by a corresponding patent filing strategy. MAbs. 2014; 6(4): 820-837, doi: 10.4161/mabs.29105, indexed in Pubmed: 24866199.

3. Bonilla-Abadía F, Coronel Restrepo N, Tobón GJ, et al. Rituximab for remission induction and maintenance in refractory systemic lupus erythematosus. Autoimmune Dis. 2014; 2014: 731806, doi: 10.1155/2014/731806, indexed in Pubmed: 24527208.

4. Van Allen EM, Miyake T, Gunn N, et al. Off-label use of rituximab in a multipayer insurance system. J Oncol Pract. 2011; 7(2): 76-79, doi: 10.1200/JOP.2010.000042, indexed in Pubmed: 21731512.

5. Kang HJ, Park JS, Kim DW, et al. Adverse pulmonary reactions associated with the use of monoclonal antibodies in cancer patients. Respir Med. 2012; 106(3): 443-450, doi: 10.1016/j. rmed.2011.11.009, indexed in Pubmed: 22154502.

6. Bitzan M, Anselmo M, Carpineta L. Rituximab (B-cell depleting antibody) associated lung injury (RALI): a pediatric case and systematic review of the literature. Pediatr Pulmonol. 2009; 44(9): 922-934, doi: 10.1002/ppul.20864, indexed in Pubmed: 19681063.

7. Biehn SE, Kirk D, Rivera MP, et al. Bronchiolitis obliterans with organizing pneumonia after rituximab therapy for non -Hodgkin's lymphoma. Hematol Oncol. 2006; 24(4): 234-237, doi: 10.1002/hon.799, indexed in Pubmed: 16948177.
8. Tonelli AR, Lottenberg R, Allan RW, et al. Rituximab-induced hypersensitivity pneumonitis. Respiration. 2009; 78(2): 225229, doi: 10.1159/000163069, indexed in Pubmed: 18843175.

9. Heresi GA, Farver CF, Stoller JK. Interstitial pneumonitis and alveolar hemorrhage complicating use of rituximab: case report and review of the literature. Respiration. 2008; 76(4): 449453, doi: 10.1159/000104866, indexed in Pubmed: 17596682.

10. Farhat L, Dara J, Duberstein S, et al. Secondary Hypogammaglobulinemia After Rituximab for Neuromyelitis Optica: A Case Report. Drug Saf Case Rep. 2018; 5(1): 22, doi: 10.1007/ s40800-018-0087-y, indexed in Pubmed: 29752554.

11. Diaz JC, Vallejo S, Cañas CA. Drug-induced lupus in anti-TNF-alpha therapy and its treatment with rituximab. Rheumatol Int. 2012; 32(10): 3315-3317, doi: 10.1007/s00296-011-2137-y, indexed in Pubmed: 21881980.

12. Barker AF. Bronchiectasis. N Engl J Med. 2002; 346(18): 13831393, doi: 10.1056/NEJMra012519, indexed in Pubmed: 11986413.

13. Ramakrishnan VR, Ferril GR, Suh JD, et al. Upper and lower airways associations in patients with chronic rhinosinusitis and bronchiectasis. Int Forum Allergy Rhinol. 2013; 3(11): 921927, doi: 10.1002/alr.21204, indexed in Pubmed: 23881553.

14. King P, Holdsworth S, Freezer $\mathrm{N}$, et al. Bronchiectasis. Intern Med J. 2006; 36(11): 729-737, doi: 10.1111/j. 1445-5994.2006.01219.x, indexed in Pubmed: 17040360.

15. Solanki T, Neville E. Bronchiectasis and rheumatoid disease: is there an association? Br J Rheumatol. 1992; 31(10): 691-693, indexed in Pubmed: 1393376.

16. Bamji A, Cooke N, Bamji A, et al. Association between rheumatoid arthritis and chronic bronchial suppuration. Ann Rheum Dis. 1984; 43(6): 856-21, indexed in Pubmed: 6524987.

17. McMahon MJ, Swinson DR, Shettar S, et al. Bronchiectasis and rheumatoid arthritis: a clinical study. Ann Rheum Dis. 1993; 52(11): 776-779, indexed in Pubmed: 8250608.

18. Shadick NA, Fanta CH, Weinblatt ME, et al. Bronchiectasis. A late feature of severe rheumatoid arthritis. Medicine (Baltimore). 1994; 73(3): 161-170, indexed in Pubmed: 8190039.

19. Chung SD, Chen PY, Lin HC, et al. Comorbidity profile of chronic rhinosinusitis: a population-based study. Laryngoscope. 2014; 124(7): 1536-1541, doi: 10.1002/lary.24581, indexed in Pubmed: 24395611.

20. Cooper N, Arnold DM. The effect of rituximab on humoral and cell mediated immunity and infection in the treatment of autoimmune diseases. Br J Haematol. 2010; 149(1): 3-13, doi: 10.1111/j. 1365-2141.2010.08076.x, indexed in Pubmed: 20151975. 